\title{
Breve argumentación didáctica de la mecánica cuántica de muchos cuerpos
}

\section{Brief didactic argumentation on quantum mechanics of many bodies}

\section{Breve argumentação didática da mecânica quântica de muitos corpos}

\author{
Cristian Andrés Aguirre-Téllez ${ }^{1}$, José Barba-Ortega ${ }^{2}$
}

Forma de citar: C.A. Aguirre-Téllez y J. Barba-Ortega, "Breve argumentación didáctica de la mecánica cuántica de muchos cuerpos", Respuestas, vol. 22, no. 1, pp. 29-36, 2017.

Recibido:

Agosto 22 de 2016

Aceptado:

Noviembre 30 de

2016

${ }^{1}$ Magister en Física caaguirret@unal.edu.co Orcid: 0000-0002-8643-1383

Departamento de Física

Universidad Nacional de

Colombia

Bogotá-Colombia

${ }^{2}$ Doctor en Física jjbarbao@unal.edu.co Orcid: 0000-0003-3415-1811

Departamento de Física

Universidad Nacional de

Colombia

Bogotá-Colombia

\section{Resumen}

El problema general en mecánica cuántica está basado en la solución de una ecuación en valores propios de un operador dado (en una representación adecuada), generalmente dicho operador es el Hamiltoniano que da cuenta de la interacción energética (salvo que dependa del tiempo) del sistema en cuestión. La solución de la ecuación de Schrödinger permite escribir el comportamiento dinámico del sistema sometido a ciertas restricciones. Sin embargo, la solución analítica de esta ecuación es viable solo en sistemas simples, cuando el sistema se describe desde la interacción de muchas partículas (problema electrónico-base de la construcción de sistemas cuánticos complejos aplicable a la descripción de moléculas, sólidos y sistemas cuánticos interactuantes en general.) la solución de la ecuación de Schrödinger del sistema no se puede realizar vía método analítico; con lo cual existe una forma más global de enfrentar dicho problema, el método auto consistente; mediante el cual se puede solucionar sistemas complejos de muchos cuerpos. Es así que en el presente paper presentamos una comparación entre el sistema auto consistente y algunas variantes que existen, con el método analítico en sistemas de muchos cuerpos y como opera dicho método, esto aplicado a un problema de dos cuerpos con interacción Coulombiana, ya que este problema presenta solución analítica y ha sido extensamente estudiado; esto con la finalidad de que los estudiantes interesados en la materia comprendan como se abordan problemas vía métodos auto consistentes y como opera este método, ya que en la literatura pocas veces se presenta el algoritmo de solución mediante este método.

Palabras clave: Mecanica Cuantica, metodo auto-consistente, problema de dos cuerpos.

\begin{abstract}
The general issue in quantum mechanics is based on the solution of an equation in eigenvalues of a given operator (in a suitable representation), generally such operator is the Hamiltonian that accounts for the energy interaction (unless it depends on the time) of the system in question. The solution of the Schrodinger equation allows writing the dynamic behavior of the system subject to certain restrictions. however, the analytical solution of this equation is feasible only in simple systems, when the system is described from the interaction of many particles (electronic problem- basis of the construction
\end{abstract}


of complex quantum systems applicable to the description of molecules, solids and interacting quantum systems in general.), the solution of the Schrödinger equation of the system cannot be performed via analytical method; therefore, there is a more global way of facing this problem, the self-consistent method; through which complex systems of many bodies can be solved. Thus, in the present paper we present a comparison between the self-consistent system and some other existent variants, with the analytical method in systems of many bodies and how this method operates, this applied to a problem of two bodies with Colombian interaction, given the analytical solution presented in this problem that has been extensively studied; this in order that students interested in the subject understand how problems are addressed through self-consistent methods and how this method operates, since in the literature the solution algorithm is rarely presented using this method.

Keywords: Quantum mechanics, Self consistent field, two body problem.

\section{Resumo}

O problema geral em mecânica quântica está baseado na solução de uma equação em valores próprios de um operador determinado (numa representação adequada), geralmente dito operador é o Hamiltoniano que dá conta da interação energética (a menos que dependa do tempo) do sistema em questão. A solução da equação de Schroedinger permite escrever o comportamento dinâmico do sistema sometido a certas restrições. No entanto, a solução analítica desta equação é viável só em sistemas simples, quando o sistema se descreve desde a interação de muitas partículas (problema electrónico-base da construção de sistemas quânticos complexos aplicável à descrição de moléculas, sólidos e sistemas quânticos que geralmente interagem) a solução da equação de Schroedinger do sistema não pode realizar-se via método analítico; com o qual existe uma forma mais global de enfrentar dito problema, o método auto consistente; através do qual se pode solucionar sistemas complexos de muitos corpos. Neste estudo, apresentamos uma comparação entre o sistema auto consistente e algumas variantes que existem, com o método analítico em sistemas de muitos corpos e como é a operação de dito método, isto aplicado a um problema de dois corpos com interação Coulombiana, já que este problema apresenta solução analítica e tem sido extensamente estudado; isto com a finalidade de que os alunos interessados na disciplina compreendam como se abordam problemas via métodos auto consistentes e a operação deste método, já que na literatura poucas vezes se apresenta o algoritmo de solução através deste método.

Palavras-chave: Mecânica quântica, método auto consistente, problema de dois corpos.

\section{Introducción}

La generalización, para muchos cuerpos, a partir de los postulados de la mecánica cuántica, se establece desde la forma inicial de la ecuación de Schrödinger, la cual es la ecuación base para la solución de todos los problemas, que pertenezcan al régimen cuántico [1], [9]. Esta ecuación (1), presenta todas las cualidades del sistema a evaluar, $\mathrm{y}$ todas las propiedades que intervengan, externas o internas a dicho sistema.

$\hat{H} \Psi=\mathrm{E} \Psi$

Aquí $\hat{H}$, es el operador de Hamilton, o Hamiltoniano, este establece las propiedades del sistema a estudiar, $\Psi$ es la función que define el estado cuántico, en la base de la 
posiciones. El valor $\mathrm{E}^{1}$, es el de la energía propia, este es el valor adquirido por la medición de una cantidad dinámica en dicho sistema. Sin embargo, el hecho de abordar diferentes sistemas, implica que la forma matemática de la ecuación de Schrödinger adquirirá formas mucho más complejas. Con ello, se llega a la generalización de problemas de muchos cuerpos.

Por medio de la generalización del problema a $n$, cuerpos se establece el Hamiltoniano electrónico [1], [2], [3], [4], [5], en el cual se establecen todas las interacciones para el sistema a estudiar, la dificultad radica, en que el Hamiltoniano no es separable en ningún sistema de coordenadas conocido [4], por lo cual la solución analítica no es aplicable en problemas de $n$ cuerpo, este Hamiltoniano posee la siguiente forma (2).

$\hat{H}_{e}=-\sum_{i}^{n} \frac{\nabla_{i}^{2}}{2}+\sum_{A}^{m} \frac{-\nabla_{A}^{2}}{2}-\sum_{i, A}^{n, m} \frac{Z_{A}}{\left|r_{i}-R_{A}\right|}+\sum_{\substack{i, j \\ i \succ j}}^{n, m} \frac{1}{\left|r_{i}-r_{j}\right|}$

$+\sum_{\substack{A, B \\ A \succ B}}^{m, m} \frac{1}{\left|r_{A}-r_{B}\right|}$

En este operador intervienen la energía cinética de todas las partículas y de los núcleos que componen la estructura a estudiar, primer y segundo término de la igualdad, el tercer, cuarto y quinto termino, establece la interacción tipo Coulomb entre los núcleoselectrones, electrones-electrones y núcleosnúcleos, son estos términos los que impiden la separación de dicho Hamiltoniano.

La solución de la ecuación de valores propios, a la cual pertenece este operador establece la solución al problema de $n$ cuerpos [1], para dicha solución, se han generado varios métodos, siendo los dos principales el analítico y el Auto-Consistente, la diferencia entre ambos, es su forma operativa. El desarrollo de los métodos son variados, y con ello, para

${ }^{1}$ Siempre y cuando, sea para la ecuación de Schrödinger estacionaria. el tratamiento vía método Auto-Consistentes, existen gran cantidad de variantes que hacen referencia a la función usada como inicial [2], [6], [7], si es o no superposición de alguna forma especial de un tipo estándar de función, o si en ella hay o no constantes que se establecen a partir de la experimentación [6].

Los métodos analíticos, ya se establecen en textos referentes a estos temas, sin embargo los métodos Auto-Consistentes, que son ampliamente usados y referenciados no se les confiere la misma importancia. Con ello concretar la calidad aplicativa de los métodos $\mathrm{SCF}^{2}$ e inferir la forma de proceder, es esbozado en los siguientes párrafos.

Con ello, se establecerá un acercamiento a los métodos vía analítica y Auto-Consistente, para un problema concreto, el problema de 2 cuerpos con interacción tipo Coulombiana.

\section{Solución en mecánica cuántica de muchos Cuerpos.}

La solución de la ecuación de Schrödinger (3), con el operador electrónico, permite la solución al problema de $n$ cuerpos. Con la aplicación del operador de Hamilton, en términos de la ecuación de Schrödinger, se obtiene la ecuación de Hartree-Fock, la cual en esencia es la base para la solución del sistema que se esté evaluando. Dado esto, se establece el operador de Hartree-Fock.

$$
\hat{H}_{f}=\hat{h}_{n}+\sum_{a} \hat{C}_{a}-\hat{K}_{a}
$$

En este operador la expresión $h_{n}$, hace referencia a la energía cinética y el potencial
Enero - Junio 2017 ISSN 0122-820X

E-ISSN 2422-5053

PP: 29-36 para la n-ésima partícula, $\hat{C}_{a}$ es el operador de Coulomb y $\hat{K}_{a}$ es el operador de intercambio, donde todos estos operadores se encuentran representados en la base de las posiciones. Clásicamente el operador de Coulomb posee una analogía directa, como la densidad del potencial experimentada por la n-ésima

\footnotetext{
${ }^{2}$ Self-Consistent Field
} 
No. 1

Enero - Junio 2017 ISSN 0122-820X E-ISSN 2422-5053 PP: 29-36 partícula, como consecuencia de las (n-1) partículas restantes [8], [9], [10]. Pero, el operador de intercambio, está fuera de poseer una analogía en este régimen clásico, siendo el régimen de la mecánica cuántica de muchos cuerpos su único campo de acción. Este operador posee una forma integro-diferencial, la cual se determinara al abordar el problema específico de dos cuerpos bajo interacción Coulombiana.

Es evidente, que la solución al problema electrónico no es simple, por ello resulta más útil transformar la ecuación integro-diferencial resultante, en una ecuación más tratable, como lo es una ecuación matricial, este proceso se conoce como método de HartreeFock-Roothaan [1]. Este se ejecuta por medio de procesos matemáticos, que se visualizan desde la perspectiva variacional del problema físico, es decir, se establece la minimización de la energía del sistema, con ello bajo la minimización del Hamiltoniano electrónico se llega a la ecuación de Hartree-Fock, y luego aplicando la idea de la completitud de las funciones de onda nos con lleva a la ecuación de Hartree-Fock-Roothaan[1],[3], que es una ecuación matricial, la cual es más simple de solucionar, que su forma integródiferencial (4):

$$
H C=\varepsilon S C
$$

Aquí, $\mathrm{H}$ representa la matriz del operador de Hamilton, $\mathrm{C}$ es una matriz de constantes, la cual se escoge para realizar la transformación del operador de Hamilton, S es la matriz métrica, para la cual su determinante posee valores entre cero y $1,0 \leq \operatorname{det}(S) \leq 1$, por ello se le designa como matriz métrica, aunque a veces cambia, adquiriendo valores intermedios, dependiendo del sistema a tratar, en general esto sucede porque si la base es una superposición infinita de términos, cumple la condición de orto- normalización, pero al momento de solucionar el problema, esta superposición se debe truncar y por ello la orto-normalización no es garantizada [10], [11] y $\quad \varepsilon$ es el valor propio para el operador de Hamilton, con la solución de esta ecuación matricial, se establece la solución al problema electrónico. El proceso vía Auto-Consistente se aborda al solucionar la ecuación de valores propios, con lo cual, se establece para el problema de 2 cuerpos.

\section{Problema de 2 cuerpos bajo interacción Tipo Coulomb}

El problema de 2 cuerpos bajo interacción Colombiana se aborda, porque se poseen resultados de la aplicación de varios métodos, siendo el analítico uno de ellos y se resalta que la solución de problemas en mecánica cuántica de muchos cuerpos, por vía analítica en pocas ocasiones es posible, por ello es tácitamente viable la comparación directa de los resultados obtenidos por varios métodos, facilidad que posee este problema, frente a otros de mayor complejidad. Establezcamos el operador de Hamilton (5) en la base de las posiciones para dicho sistema.

$$
\hat{H}=-\frac{\nabla^{2}{ }_{i}}{2}-\frac{\nabla^{2}{ }_{j}}{2}-\frac{1}{\left|r_{i}-R_{A}\right|}-\frac{1}{\left|r_{j}-R_{b}\right|}+\frac{1}{\left|r_{i}-r_{j}\right|}
$$

Este es el operador asociado al problema de 2 electrones bajo interacción Coulombiana con el núcleo que suponemos estático (aproximación adiabática), y entre ambos electrones; las unidades establecidas para este operador son las unidades de Hartree, por comodidad en el tratamiento [1],[3].

Luego de la igualación de dicho operador en la ecuación de Schrödinger, se obtiene el operador de Hartree-Fock, pero antes, se debe establecer que siempre hay una función determinada para el estado base del sistema a estudiar, esta función para dicho estado base, o de mínima energía, se encuentra a través del determinante de Slater, el cual, nos proporciona la forma de la ecuación de onda, para dicho estado, la cual debe cumplir el principio de 
anti-simetría de las funciones de onda [1], [2]. Este resultado es de basta importancia, ya que establece que la permutación de partículas idénticas, que son indistinguibles ${ }^{3}$, le altera el signo a la función de onda (6), esto es como intercambiar el sistema de coordenadas de las partículas [10]-[12].

$$
\Psi=\frac{1}{\sqrt{N !}}\left|\begin{array}{cccc}
\psi_{i}\left(x_{1}\right) & \psi_{j}\left(x_{2}\right) & \ldots . & \psi_{k}\left(x_{1}\right) \\
\psi_{i}\left(x_{2}\right) & \psi_{j}\left(x_{2}\right) & \ldots . & \psi_{k}\left(x_{2}\right) \\
\ldots . & \ldots . & \ldots . & \ldots . \\
\psi_{i}\left(x_{n}\right) & \psi_{j}\left(x_{n}\right) & \ldots . & \psi_{k}\left(x_{n}\right)
\end{array}\right|
$$

Aquí la función de onda cumple la antisimetría, caso que queda comprobado por el intercambio de filas, con lo cual el signo de dicho determinante cambia, además, se aclara que las $x_{n}$ representan no solo la cualidad posicional del sistema considerado, sino, que también la propiedad de espín, como caso particular este determinante, queda reducido a un determinante de $2 \times 2$, para el sistema considerado, aunque la forma de la función, no se especifique de forma alguna, esto se verá reflejado en el cálculo Auto-Consistente. Luego de la igualación, de la función de onda, para el estado base, y el operador Hamiltoniano que describe el sistema a estudiar, se llega a la ecuación de Hartree-Fock en forma integrodiferencial (7).

$$
\begin{aligned}
& \left.\hat{h}_{1}(1) x_{a}(1)+\sum_{b \neq a} \mid \int x_{b}{ }^{*}(2) \hat{r}_{b}(2) d x_{2}\right] x_{a}(1)- \\
& \sum_{b \neq a}\left[\int x_{b}{ }^{*}(2) x_{a}(1) \hat{r} d x_{2}\right] x_{b}(1)=\varepsilon_{a} x_{a}(1)
\end{aligned}
$$

Las funciones, se representan por las $x$ 's, la pertenencia a los cuerpos se destaca por medio de los paréntesis, es decir, la función $x_{a}(1)$ es la función para la partícula $a$, enumerada como la partícula 1, el operador $\hat{r}$, es el operador inverso a la distancia nuclear, $\hat{h}_{1}$

${ }^{3}$ Esta indistingibilidad surge de la falta de trayectoria en mecánica cuántica como ya se menciono es la suma del operador de energía cinética más la potencial de la partícula enumerada por su paréntesis, $\varepsilon_{a}$ es la energía propia, resultado de la aplicación del operador de Hartree-Fock. Se ha dejado explícitos algunos términos en paréntesis cuadrados, con el objetivo de visualizar los operadores de Coulomb, y el de intercambio, primer y segundo paréntesis cuadrado respectivamente.

Por medio de un proceso, tal como se mencionó se pude transformar esta ecuación integro-diferencial, en matricial, quedando al ecuación de Hartree-Fock-Roothaan, Sin embargo el proceso por el método AutoConsistente, posee una direccionalidad definida de la siguiente forma. Se establece una función inicial, de la cual depende la calidad de los datos arrojados para los valores propios, casi siempre se establece como una función tipo Gauss (GTO) o Tipo Slater (STO) (8), dependiendo de la rigurosidad o la aproximación que se desee establecer [13][15].

$$
\begin{aligned}
& \phi_{S}=\left(\frac{\zeta}{\pi}\right)^{\frac{1}{2}} \exp -\zeta\left(\left|r-R_{a}\right|\right) \\
& \phi_{G}=\left(\frac{2 \alpha}{\pi}\right)^{\frac{3}{4}} \exp -\alpha\left(\left|r-R_{a}\right|\right)^{2}
\end{aligned}
$$

Los coeficientes, $\zeta$ y $\alpha$ deben escogerse de acuerdo a la maximización de ciertas cantidades (energía y $\operatorname{Det}(\mathrm{S})$ ), esta elección también establece diferencias entre el proceder mismo del método SCF, es más hay variantes para las cuales los nombres establecidos dejan claras las funciones iniciales usadas. Luego se establecen las cantidades que se deseen encontrar, para poder estructurar el operador de Fock, estas cantidades son los valores esperados para la energía cinética, potencial e integrales electrónicas; siendo las ultimas las más complejas, dado su desarrollo matemático, y la cantidad necesaria de iteraciones usadas
Enero - Junio 2017 ISSN 0122-820X E-ISSN 2422-5053

PP: 29-36 
No. 1

Enero - Junio 2017 ISSN 0122-820X E-ISSN 2422-5053 PP: 29-36 para hallar su convergencia, estas integrales dobles son resultados de los operadores de intercambio y Coulomb, por lo cual casi siempre se usan funciones tipo Gauss, por su facilidad operativa, y además estas mejoran en gran medida los pasos matemáticos, la integral electrónica, posee la forma (9).

$$
G=\iint_{r_{1}, r_{2}} \phi_{\mu}^{*}{ }_{\mu}(1) \phi_{v}(2) \hat{r} \phi^{*}{ }_{\lambda}(2) \phi_{\sigma}(2) d r_{1} d r_{2}
$$

A continuación se establece una tabla con valores obtenidos por diferentes métodos, entre ellos algunas variantes del método de Hartree-Fock-Roothaan [9]-[11], el método analítico M.A, experimental y el respectivo porcentaje de aproximación, en comparación con los valores hallados por el método experimental.

\section{Cálculos y comparación realizada}

Bajo las consideraciones realizadas para el método auto consistente, solucionaremos la ecuación de Hartree-Fock-Roothaan con el Hamiltoniano electrónico presentado para el problema de dos cuerpos interactuando con un potencial Coulombiano. Estos datos serán correlacionados con los datos obtenidos por medios experimentales, para lo cual tomamos como referencia [8]-[10] que establece la energía del estado base para el sistema de 2 electrones como (unidades de Hartree) [14]. Adicional, en el caso del método auto consistente STO (Slater Type Orbital), se tomó, para el caso de 3G (Gauss type Orbital), como superposición de tres términos lineales (10).

$$
\phi_{3 G}=\sum_{i=1}^{3}\left(\frac{2 \alpha}{\pi}\right)^{\frac{3}{4}} \exp -\alpha\left(\left|r_{i}-R_{a}\right|\right)^{2}
$$

Siendo, la posición de cada uno de los electrones y $\mathrm{R}_{\mathrm{a}}$ la posición del núcleo del sistema, los valores de $\alpha$ se toman tal que al realizar una cantidad de iteraciones se minimice o la energía del estado base, o que la función converja a cierto valor. Para el sistema tomado como solución la función DZ (Dunning-Huzinaga-Duijneveldt), dos funciones STO (con diferentes exponentes, $\zeta$ ) para cada capa interna y una para cada orbital atómico en la capa de valencia. Bajo esta caracterización y datos iniciales, se presenta la tabla I, que da cuenta de los datos obtenidos para el problema específico tratado.

Tabla I. Comparación de valores obtenidos para la energía del sistema de 2 electrones bajo interacción Coulombiana, por diferentes métodos

\begin{tabular}{|c|l|l|l|l|}
\hline M.A[8] & SCF -STO & SCF-3G & SCF-DZ & EX [3], \\
\hline 4.003 & 3.8502 & 3.9673 & 3.99085 & 4.016 \\
\hline $99.676 \%$ & $95.871 \%$ & $98.787 \%$ & $99.373 \%$ & $100 \%$ \\
\hline
\end{tabular}

Fuente: Autores

En la tabla I Comparación de valores obtenidos para la energía del sistema de 2 electrones bajo interacción Coulombiana, por diferentes métodos M.A (método analítico), SCF-STO (Auto consistente - Slater type orbital), SCF-3G (Auto consistente - 3 Gauss Type Orbital), SFC-DZ (Auto consistente - Doble zeta o Dunning Huzinaga) y EX (Energía Experimental)
Estos datos, dan una visión bastante extensa sobre la calidad en las aproximaciones de cada uno de estos métodos, por ello, se establecen las principales diferencias entre el método analítico y el método Auto-Consistente con sus respectivas ventajas y desventajas (Tabla II y III), ya que esto ayuda a una mejor visualización, pedagógicamente hablando, ya que el objetivo principal es brindar una forma didáctica de comparación. 
Tabla II. Ventajas y desventajas -método Analítico-

\begin{tabular}{|c|c|}
\hline \multicolumn{2}{|c|}{ Método analítico } \\
\hline Ventajas & Desventajas \\
\hline $\begin{array}{c}\text { solución exacta a los problemas plantea- } \\
\text { dos }\end{array}$ & $\begin{array}{c}\text { Carencia de generalidad en la solu- } \\
\text { ción de problemas }\end{array}$ \\
\hline $\begin{array}{c}\text { Ejecuciones de problemas que pueden ser } \\
\text { abordados desde muchos campos }\end{array}$ & $\begin{array}{c}\text { Algunas veces con el aumento de la } \\
\text { dificultad del sistema, es inaplicable } \\
\text { este método }\end{array}$ \\
\hline $\begin{array}{c}\text { Aplicabilidad directa al problema en } \\
\text { cuestión }\end{array}$ & $\begin{array}{c}\text { Demasiadas extensiones para la } \\
\text { solución de un sistema particular }\end{array}$ \\
\hline $\begin{array}{c}\text { Solo son necesarios conocimientos } \\
\text { matemáticos para hallar dichas solucio- } \\
\text { nes. }\end{array}$ & Es necesario gran conocimiento \\
& matemático \\
& \\
\hline
\end{tabular}

Fuente: Autores

Tabla III Ventajas y desventajas -método Auto-Consistente-

\begin{tabular}{|c|c|}
\hline \multicolumn{2}{|c|}{ Método Auto-Consistente } \\
\hline Ventajas & Desventajas \\
\hline $\begin{array}{c}\text { Generalidad de solución, sin implicación } \\
\text { directa del sistema a evaluar }\end{array}$ & $\begin{array}{c}\text { Establecimiento de una función de } \\
\text { arranque, de la cual depende en su } \\
\text { gran mayoría la calidad de datos } \\
\text { obtenidos }\end{array}$ \\
\hline $\begin{array}{c}\text { Si se establecen buenas funciones iniciales } \\
\text { y un número considerable de iteraciones } \\
\text { arroja excelentes valores }\end{array}$ & $\begin{array}{c}\text { Para agilizar soluciones es preferi- } \\
\text { ble realizar rutinas de programa- } \\
\text { ción con lo cual, es necesario } \\
\text { poseer conocimientos de progra- } \\
\text { mación }\end{array}$ \\
$\begin{array}{c}\text { Evaluación rápida y precisa de cantidades } \\
\text { que se deseen. }\end{array}$ & $\begin{array}{c}\text { Aunque se obtenga resultados } \\
\text { estos no dejan de ser aproximación } \\
\text { de los datos exactos }\end{array}$ \\
\hline $\begin{array}{c}\text { Gran variedad de métodos, para aplicar a } \\
\text { dicho sistema, con lo cual se garantiza que } \\
\text { el sistema por alguno de dichos métodos } \\
\text { posea solución. }\end{array}$ & $\begin{array}{c}\text { Contar con la maquina adecuada } \\
\text { para la realización de extensos } \\
\text { cálculos en forma iterativa. }\end{array}$ \\
\hline
\end{tabular}

Fuente: Autores
Vol. 22

No. 1

Enero - Junio 2017 ISSN 0122-820X

E-ISSN 2422-5053

PP: 29-36

\section{Conclusiones}

En los sistemas mecánico cuánticos de muchos cuerpos, la solución de sistemas de $n$ cuerpos es generalmente no soluble por métodos analíticos, por lo cual se establecen métodos auto consistentes para dichas soluciones. En el presente trabajo se realizó un desarrollo teórico de la teoría Hartree Fock- Roothaan, con lo cual se estableció la solución de un problema específico (problema de dos cuerpos con interacción Coulombiana), mediante el hamiltoniano electrónico en la base de las posiciones. Así pues, presentamos la comparación de datos obtenidos para la energía del estado base mediante el método experimental, tres variantes del método auto consistente STO, 3-GTO y DZ, que son en general los sistemas más utilizados en cálculos de auto consistencia. En la tabla I se presentaron dichas comparaciones dados los valores obtenidos por los diferentes métodos, se hace evidente que la exactitud de estos métodos auto consistentes son superiores al $90 \%$, incluso en algunos de ellos (DZ), tienen exactitud comparables al método analítico. 
Vol. 22

No. 1

Enero - Junio 2017

ISSN 0122-820X

E-ISSN 2422-5053

PP: 29-36
En la tabla II y III se presentan las ventajas y desventajas de los métodos analíticos y auto consistentes, esto presenta una visión más general de cómo operan ambos métodos y pedagógicamente hablando, establece una estructura para la aplicación de cada uno de los métodos, que es una herramienta de gran utilidad para aquellas personas que están interesadas en la solución de problemas en química cuántica.

\section{Referencias}

[1] A. Szabo and N. Ostlund, "Modern Quantum Chemistry", Dover, New York, 1989.

[2] I. Levine, "Química Cuántica”, Prentice Hill, Madrid, 2001.

[3] L. Errol, "Computacional Chemistry", Kluwer Academia Publishers, New York, 1995.

[4] F. Jensen, "Introduction to Computacional Chemistry", Jhon wiley and Sons, pp. 122-126, 2007.

[5] J. Cramer, "Essentials of computational Chemistry", Jhon wiley and Sons, pp. 17-19, 2002.

[6] C. Pekeris, "Ground States of twoelectrons atoms". Phys Rev, vol. 112, no. 5, pp. 1649-1658, 1958.

[7] E. Clementy, "Tables of Atomic Functions", International Business Machines Corp, pp. 2-9, 1965.

[8] C. Froese, "The Hartree-Fock Method for Atoms: A Numerical Approach", John Wiley \& Sons Inc, pp. 28-86, 1997.

[9] L. Landau, "Mecánica Cuántica NoRelativista", Ed Mir, Moscú, 1985.
[10] A. Nussbaum, "Applied Group Theory for Chemists", Physicists and Engineers, pp. 01-103, 2010.

[11] J.C Slater, "A Simplification of the Hartree-Fock Method", Phys. Rev, vol. 81, no. 3, pp. 385-390, 1951

[12] T.A. Kaplan and W.H. Kleine, "HartreeFock Theory: Slater Determinants of Minimum Energy", Phys. Rev. vol 156, no. 1, pp. 1-10, 1967.

[13] G.A Burton and J.W Thom, "Holomorphic Hartree-Fock Theory: An Inherently Multireference Approach", J. Chem. Theory Comput, vol. 12, no. 1, pp. 167-173, 2016.

[14] A. Beckel, "A new mixing of HartreeFock and local density-functional theories", J. Chem. Phys, vol. 98, no. 2, pp. 17-21, 1998.

[15] C.R Santos, C. Lobato, S. Braga, et al, "Application of Hartree-Fock Method for Modeling of Bioactive Molecules Using SAR and QSPR", Computational Molecular Bioscience, vol. 4, no.1, pp. 1-24, 2014. 